\title{
Tomographic Field Free Line Magnetic Particle Imaging With an Open-Sided Scanner Configuration
}

\author{
Can Barış Top ${ }^{\circledR}$, Member, IEEE, and Alper Güngör ${ }^{(\mathbb{})}$, Graduate Student Member, IEEE
}

\begin{abstract}
Superparamagnetic iron oxide nanoparticles (SPIONs) have a high potential for use in clinical diagnostic and therapeutic applications. In vivo distribution of SPIONs can be imaged with the Magnetic Particle Imaging (MPI) method, which uses an inhomogeneous magnetic field with a field free region (FFR). The spatial distribution of the SPIONs are obtained by scanning the FFR inside the field of view (FOV) and sensing SPION related magnetic field disturbance. MPI magnets can be configured to generate a field free point (FFP), or a field free line (FFL) to scan the FOV. FFL scanners provide more sensitivity, and are also more suitable for scanning large regions compared to FFP scanners. Interventional procedures will benefit greatly from FFL based open magnet configurations. Here, we present the first open-sided MPI system that can electronically scan the FOV with an FFL to generate tomographic MPI images. Magnetic field measurements show that FFL can be rotated electronically in the horizontal plane and translated in three dimensions to generate 3D MPI images. Using the developed scanner, we obtained 2D images of dot and cylinder phantoms with varying iron concentrations between $11 \mu \mathrm{g} / \mathrm{ml}$ and $770 \mu \mathrm{g} / \mathrm{ml}$. We used a measurement based system matrix image reconstruction method that minimizes $\ell_{1}$-norm and total variation in the images. Furthermore, we present 2D imaging results of two $4 \mathrm{~mm}$-diameter vessel phantoms with $0 \%$ and $75 \%$ stenosis. The experiments show high quality imaging results with a resolution down to $2.5 \mathrm{~mm}$ for a relatively low gradient field of $0.6 \mathrm{~T} / \mathrm{m}$.

Index Terms-Magnetic particle imaging, field free line, open magnetic particle imaging scanner, tomography, magnetic nanoparticles.
\end{abstract}

\section{INTRODUCTION}

C UPERPARAMAGNETIC iron oxide nanoparti$\checkmark$ cles (SPIONs) have been proposed as biocompatible agents for a wide variety of biomedical applications such as magnetic resonance angiography, perfusion imaging, lymph

Manuscript received June 11, 2020; revised July 30, 2020; accepted July 31, 2020. Date of publication August 4, 2020; date of current version November 30, 2020. This work was supported in part by the Scientific and Technological Research Council of Turkey (TUBITAK) under Grant 9050103. (Corresponding author: Can Barış Top.)

Can Barış Top is with the ASELSAN Research Center, Aselsan A.Ş., 06200 Ankara, Turkey (e-mail: cbtop@aselsan.com.tr).

Alper Güngör is with the ASELSAN Research Center, Aselsan A.Ş., 06200 Ankara, Turkey, and also with the Department of Electrical and Electronics Engineering, Bilkent University, 06800 Ankara, Turkey (e-mail: agungor@aselsan.com.tr).

This article has supplementary downloadable material available at https://ieeexplore.ieee.org, provided by the authors.

Color versions of one or more of the figures in this article are available online at https://ieeexplore.ieee.org.

Digital Object Identifier 10.1109/TMI.2020.3014197 node imaging, liver and spleen imaging [1]-[3]. SPIONs conjugated with ligands can be designed to target specific cells, such as tumors [4]. Under proper alternating magnetic field conditions, SPIONs heat their surroundings, enabling hyperthermia therapy and targeted drug release [5], [6]. Furthermore, targeted multi-modality imaging and therapy is possible with nanoagent conjugated SPIONs [7], [8].

In Magnetic Resonance Imaging (MRI), SPIONs provide negative or positive contrasts depending on their size and coating properties [9], [10]. The spatial distribution of the SPIONs can also be imaged directly with the emerging Magnetic Particle Imaging (MPI) method [11]. Since biological tissues do not contribute to the MPI signal, quantitative in vivo imaging of the SPIONs is possible with MPI [12]-[14]. In MPI, an inhomogeneous magnetic field with a field free region (FFR), which is called Selection Field (SF), is generated using a magnet system. FFR can be defined as the region inside SF where SPION magnetization is mainly in the linear part of the magnetization curve. For imaging, a dynamic magnetic field (Drive Field) is applied along with the SF, and the magnetization response of the SPIONs inside the FFR are picked up generally by receive coils. The SPIONs outside the FFR are in magnetic saturation, therefore, their magnetization vectors cannot be significantly altered by the dynamic field. The field of view (FOV) is scanned with the FFR, and the SPION distribution is reconstructed using the signal model based x-space method [15], [16], measurement based system matrix method [11], [17]-[19], or a combination of both methods [20].

In vivo and phantom studies have demonstrated the potential use of MPI for a diverse range of clinical applications including: real time cardiac imaging [21]-[23]; instrument visualization for cardiovascular interventions [24]-[27]; catheter steering [28]; assessment of aneursym hydrodynamics [29]; stenosis quantification [30], [31]; cell tracking [32]-[34]; tumor imaging [35]-[37]; perfusion imaging [38]-[41]; imaging of gastrointestinal bleeding [13]; temperature imaging [42]-[44]; guided and focused hyperthermia therapy [45]; quantitative tracking of inhaled aerosols [46]; sentinel lymphnode detection [47]; imaging of cerebral blood volume [48]; and viscosity mapping [49], [50].

Although a commercial clinical MPI scanner is not yet available, studies show the feasibility of MPI systems for human imaging [51]-[53]. Moreover, a head-sized clinical 
prototype has recently been introduced for imaging of blood perfusion in the brain [54].

The FFR in MPI can be a field free point (FFP) or a field free line (FFL). As the total volume of an FFL is larger than that of an FFP, the total number of responsive particles is greater in the FFL scanners compared to the FFP scanners. Therefore, FFL scanners exhibit higher sensitivity than FFP scanners [55], [56]. They are also suitable for large imaging volumes required for clinical applications.

Closed bore FFL MPI scanners have been proposed for both projection and tomographic imaging. A fully electronic scanner for tomographic imaging was introduced by Erbe et al. [57]. Bente et al. reported imaging experiments using this configuration [58]. Similar configuration was later up-scaled to a rabbit-sized scanner [59]. Projection FFL imaging scanner configurations were proposed as well [60], [61]. Konkle et al. demostrated 3D imaging using a projection FFL scanner configuration, by rotating the sample and taking multiple projection acquisition [62], [63].

Although closed bore scanners have the advantage of high coil efficiency due to small FOV-to-coil distance, open configurations would be preferable for interventional applications. Single-sided coil configurations, in which the object is constrained from only one side, have been introduced for this purpose [64], [65]. However, in single-sided systems, the magnetic field gradient degrades as the FFR is moved away from the coils. As a result, sensitivity and resolution deteriorate with FFR depth. Therefore, single-sided configurations are particularly suitable for superficial applications.

We recently proposed an open-sided FFL scanner configuration, which is free of gradient degradation effect [52]. With this configuration, the operator can interact with the patient allowing interventional procedures. In addition, single-axis drive and receive coils are sufficient for tomographic imaging due to the orthogonality between the magnetic field and its gradient.

Here, we present the first open-sided FFL scanning MPI prototype system. We show that the FFL can be rotated to any direction, and translated electronically in the horizontal plane for 2D imaging. Furthermore, we also show that the imaging plane can be moved electronically in the out-of-plane direction by asymmetrical excitation of the upper and lower selection field coil groups. Therefore, the system is capable of 3D imaging by translating the imaging plane continuously or in discrete steps. Size of the vertical gap between the upper and lower coil groups is adjustable so that the magnetic field gradient can be maximized according to the imaged object size. We present 2D imaging results of tube phantoms filled with Perimag (Micromod, Germany) SPIONs with varying iron concentrations $(11 \mu \mathrm{g} / \mathrm{ml}$ to $770 \mu \mathrm{g} / \mathrm{ml})$; dot phantoms with different dot diameters $(2.5 \mathrm{~mm}$ to $4 \mathrm{~mm})$; and vessel phantoms with $0 \%$ and $75 \%$ stenosis, using our system.

\section{Methods}

The open-sided FFL scanner prototype components and reconstruction methods are given in this Section.

\section{A. Scanner Hardware}

1) Selection Field Generation: In the current configuration, selection field is a magnetic field with an FFL that can be

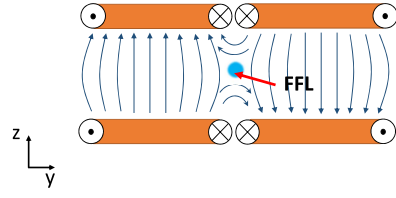

(a)

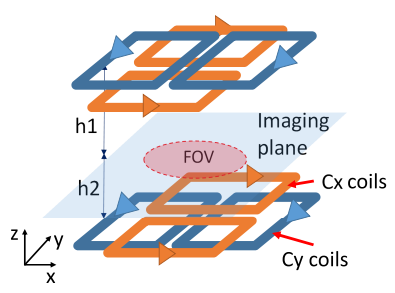

(c)

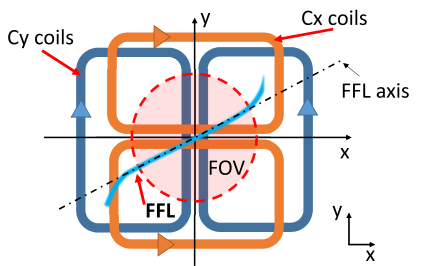

(b)

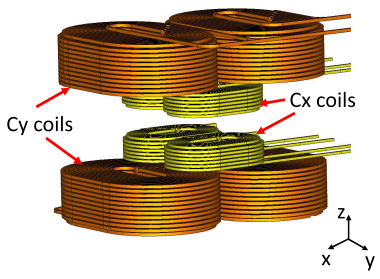

(d)
Fig. 1. Open-sided configuration for the electronically rotating FFL. (a) $C x$ coil group consisting of two coil pairs fed with alternating current directions generate an FFL along the x-direction, (b) an FFL can be generated at any arbitrary direction using two orthogonal FFL generating coil groups, $C x$ and $C y$, (c) the imaging plane in the z-direction can be steered by asymmetrical excitation of top and bottom coil groups. $h 1$ and $h 2$ are the distances between selection field coil groups and imaging plane, (d) the model of the designed $C x$ and $C y$ coil groups used in the current prototype.

rotated electronically as follows. An FFL along x-direction is generated using a biplanar gradient coil configuration (Fig. 1(a)). We denote this coil group as the $C x$ coil group. It should be noted that the magnetic field vector is in the $\mathrm{z}$-direction, whereas its gradient is in the y-direction. A y-directed FFL is generated using a coil configuration similar to the $C x$ coil group but rotated $90^{\circ}$ about the $\mathrm{z}$-axis (Fig. 1). This coil group will be denoted as the $C y$ coil group. We show in the following analysis that electrical rotation and translation of the FFL in the xy-plane is possible using $C x$ and $C y$ coil groups with the addition of an homogeneous z-directed magnetic field.

Assuming a constant gradient in the vicinity of the FFL, the total selection magnetic field $\left(\mathbf{H}_{\mathbf{S}}\right)$ in the midplane between the upper and lower coils $(z=0)$ can be written as:

$$
\begin{aligned}
\mathbf{H}_{\mathbf{S}} & =\mathbf{H}_{\mathbf{C x}}+\mathbf{H}_{\mathbf{C y}} \\
& =y G_{x} \hat{\mathbf{a}_{z}}+x G_{y} \hat{\mathbf{a}_{z}},
\end{aligned}
$$

where $\mathbf{H}_{\mathbf{C x}}$ and $\mathbf{H}_{\mathbf{C y}}$ are the magnetic fields, and $G_{x}$ and $G_{y}$ are the magnetic field gradients generated by the $C x$ and $C y$ coil groups at $z=0$ plane, respectively. $x$ and $y$ are the $\mathrm{x}$ and $y$ positions in the vicinity of the FFL. $\hat{\mathbf{a}_{z}}$ is the unit vector in the $\mathrm{z}$-direction.

The gradient magnitudes can be written as the product of the gradient efficiency term $\eta_{i=x, y}(\mathrm{~T} / \mathrm{m} / \mathrm{A})$ and the current applied to the coils $I_{x}$ and $I_{y}$ :

$$
G_{x}=\eta_{x} I_{x}, G_{y}=\eta_{y} I_{y} .
$$

The equation of a rotated and translated line can be written in the form:

$$
y \cos (\phi)=x \sin (\phi)+d .
$$

where $\phi$ is the angle of rotation with respect to the X-axis, and $d$ is the translation term. 


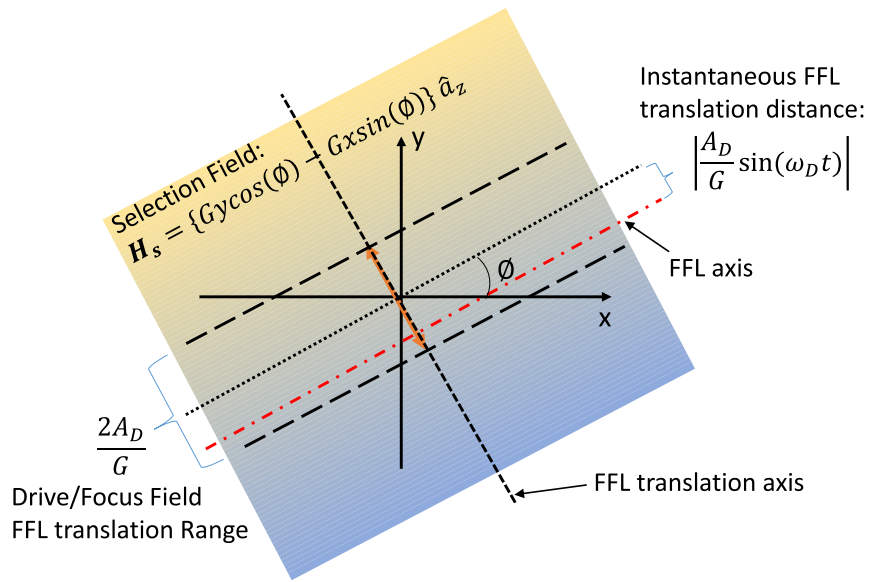

Drive/Focus Field:

$\boldsymbol{H}_{\boldsymbol{d}}=A_{D} \sin \left(\omega_{D} t\right) \hat{a}_{z}$

Fig. 2. Magnetic fields for electronic rotation and translation using the proposed coil system. The FFL translation direction is always orthogonal to the FFL axis as the selection and drive/focus fields are both in the $z$-direction, whereas the gradient is orthogonal to the FFL axis. The FFL sweeps a region of $2 A_{D} / G$.

On the FFL axis, the total field is zero. Multiplying both sides of (3) by the magnetic field gradient of the system $G$, and comparing it with (1), it is clearly seen that a rotated and translated FFL can be generated by adding a translation field $\mathbf{H}_{\mathbf{D}}=G d \hat{a}_{z}$ to the selection field $\mathbf{H}_{\mathbf{S}}$.

The expression for the SF coil gradients are then,

$$
G_{x}=-G \cos (\phi), G_{y}=G \sin (\phi),
$$

and, the total field is given by:

$$
\begin{aligned}
\mathbf{H}_{\mathbf{T}} & =\mathbf{H}_{\mathbf{S}}+\mathbf{H}_{\mathbf{D}}, \\
& =(-y G \cos (\phi)+x G \sin (\phi)+G d) \hat{\mathbf{a}_{z}} .
\end{aligned}
$$

Using (2) and (4), the selection field currents to generate an FFL with $\phi$ degrees rotation is:

$$
I_{x}=\left(-G / \eta_{x}\right) \cos (\phi), I_{y}=\left(G / \eta_{y}\right) \sin (\phi) .
$$

It should be noted that the translation field $\mathbf{H}_{D}$ is independent of the rotation angle $\phi$. As a result, unlike previous tomographic FFL scanner designs that require two separate drive (and focus) coils for electronic rotation [58], [59] it is sufficient to use single axis drive (and focus) coil(s) for 2D imaging. Figure 2 shows the geometry for FFL rotation and the extent of FFL translation due to a drive/focus field. The total scan range for a sinusoidal field $A_{D} \sin \left(\omega_{D} t\right) \hat{a}_{z}$ is $2 A_{D} / G$. For symmetrical excitation of the upper and lower coil groups (i.e. with the same current amplitudes), the FFL and the imaging plane are on the $z=0$ plane, and equidistant from the upper and lower coil groups $(h 1=h 2$ in Fig. 1(c)). Vertical position of the imaging plane can be changed by asymmetrical excitation. If the upper coil group is excited with a higher current than the lower coil group, the imaging plane moves in the $-\mathrm{z}$ direction $(h 1>h 2)$, and vice versa. By this way, vertical translation of the imaging plane allows for $3 \mathrm{D}$ imaging using a single drive field channel.

The position of the imaging plane on the z-axis can be selected by applying the following currents to the upper and lower SF coil groups:

$$
\begin{aligned}
& I_{i, \text { upper }}(z)=c_{i}(z) I_{i}\left(1+\alpha_{i}(z)\right), \\
& I_{i, \text { lower }}(z)=c_{i}(z) I_{i}\left(1-\alpha_{i}(z)\right) .
\end{aligned}
$$

TABLE I

Selection Field Coil Properties

\begin{tabular}{|l|l|l|}
\hline Parameter & Cx Coils & Cy Coils \\
\hline Number of turns & $7 \times 9$ & $12 \times 12$ \\
Conductor size $(\mathrm{mm})$ & $6 \times 6$ & $7.5 \times 7.5$ \\
Inner radius $(\mathrm{mm})$ & 2 & 2.5 \\
Resistance $(\mathrm{m} \Omega)$ & $22.0 \pm 0.1$ & $47.8 \pm 0.2$ \\
Inductance $(\mathrm{mH} @ 20 \mathrm{~Hz})$ & $0.435 \pm 0.005$ & $2.900 \pm 0.015$ \\
Outer dimensions $(\mathrm{cm})$ & $16 \times 21 \times 5$ & $25 \times 34 \times 10$ \\
\hline
\end{tabular}

In (7), $i$ is the type of the coil group ( $i=x$ for $C x, i=y$ for $C y), \alpha_{i}(z)$ is a coefficient, which is an odd function of $z$. $c_{i}(z)$ is a compensation factor to maintain constant gradient for any z-position of the imaging plane. For $h 1=h 2$, the FFL is on the $z=0$ plane and $\alpha_{i}(0)=0, c_{i}(0)=1$.

The SF coils were designed and analyzed using both an in-house developed magnetic field solver and CST EM Studio (3DS, Dassault Systemes, France). The developed solver uses Biot-Savart law to calculate the magnetic field of the coils, and was implemented in Matlab (Mathworks Inc, CA, USA). The simulation model of the coils are given in Fig. 1(d). Hollow conductors were used water cooling. The resistance and inductance of the coils were measured using E4980AL LCR meter (Keysight Technologies, CA, USA). Properties of the coils in the $C x$ and $C y$ coil groups are given in Table I.

The designed coils were manufactured by Danfysik (Denmark), and placed in a custom designed mobile mechanical housing (ANOVA R\&D technologies, Turkey). The size of the coil system is $50 \mathrm{~cm} \times 34 \mathrm{~cm} \times 29 \mathrm{~cm}$, and the total size of the system including the mechanical housing is $108 \mathrm{~cm} \times 73 \mathrm{~cm} \times 120 \mathrm{~cm}$. The mechanical housing allows adjustment of the separation between the upper and lower SF coil groups in $40 \mathrm{~mm}$ to $100 \mathrm{~mm}$ range. Therefore, the system is flexible to support imaging objects of different sizes with highest achievable gradient. The coils are driven in pairs (Fig. 3). The upper and lower $C x$ coils are driven by two FAST-PS-1K5 (Caen Els S.R.L., Italy) bipolar power supplies that can output $100 \mathrm{~A}, \pm 15 \mathrm{~V}$, and the upper and lower $C y$ coils are driven by NGPS (Caen Els S.R.L., Italy) power supplies that can output $200 \mathrm{~A}, 50 \mathrm{~V}$. The photograph of the system is shown in Fig. 4.

We measured the magnetic field and gradient properties of the coils using a Lake Shore 460 three-axis Gaussmeter, a hall effect probe (Lake Shore Cryotronics Inc., OH, USA) and a three-axis mechanical robot (Velmex Inc., NY, USA), and analyzed the magnetic field gradient along the FFL for each coil group. Moreover, we measured the gradient efficiencies $\eta_{x}$ and $\eta_{y}$ as a function of SF coil spacing.

2) Drive/Focus Field Generation: A combined drive/focus field coil generating a magnetic field in the z-direction was designed to apply the drive field and sweep the FFL in the imaging plane. We restricted the amplitude of the high frequency drive field taking peripheral nerve stimulation considerations into account. To cover the full field of view, we used the same coil to generate both a high frequency $(26 \mathrm{kHz})$ drive field and a low frequency focus field.

Since the magnetic field on the imaging plane is in the z-direction, a single-axis drive coil is sufficient to translate the FFL in the imaging plane. The drive/focus coil was designed in 


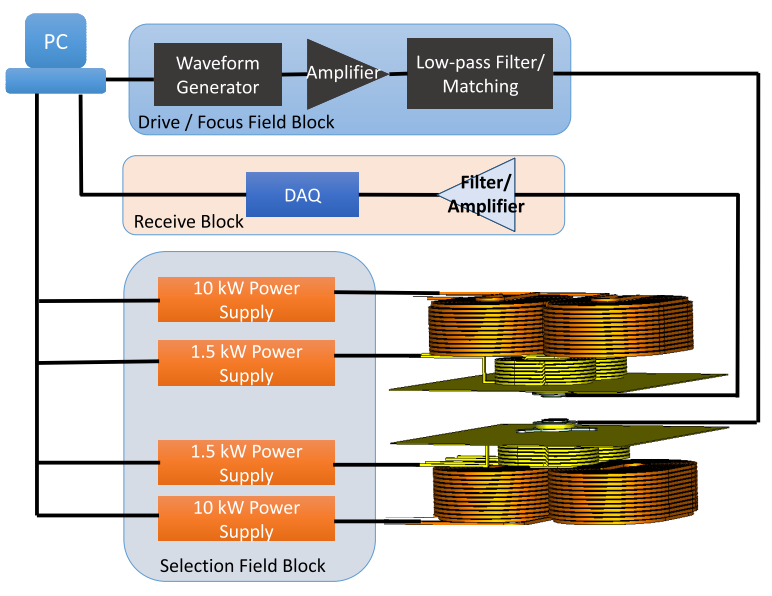

Fig. 3. The schematic diagram of the prototype system showing the selection and drive/focus field generating blocks, and the receive block.

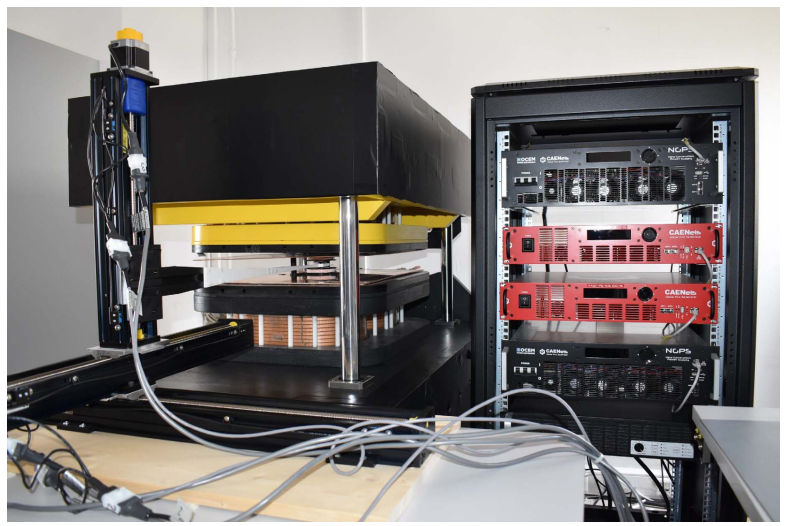

Fig. 4. The photograph of the ASELSAN open-sided FFL scanning MPI prototype system. Four DC supplies feed the eight selection coils in pairs. Size of the vertical gap between the upper and lower coil groups can be adjusted manually using a jackscrew. The drive/focus and receive coils can be replaced easily.

a Helmholtz configuration to generate a nearly homogeneous magnetic field within a circular imaging volume of $30 \mathrm{~mm}$ diameter. The inner radius, outer radius and thickness of the drive coil are $41 \mathrm{~mm}, 77 \mathrm{~mm}$ and $9 \mathrm{~mm}$, respectively. The coil has 72 turns and was wound on a 3D printed housing using a 200 strand AWG 41 litz wire (Rupalit V155, Rudolf Pack Gmbh \& Co., Germany). To isolate the high frequency magnetic field generated by the drive/focus coil from the selection field coils, $2 \mathrm{~mm}$-thick copper plates was placed between the drive/focus field coils and the selection field coils. Measured inductance and resistance of the drive coils at $26 \mathrm{kHz}$ are $0.628 \mathrm{mH}$ and $2.63 \Omega$, respectively.

A filtering and matching circuit was designed to suppress the harmonics of the AC amplifier and compensate the drive coil inductance. We used a fourth order low-pass filter to enable generation of both low frequency focus field and high frequency drive field using a single coil. The circuit elements were optimized to maximize the first to third harmonic ratio and the transductance of the circuit at $26 \mathrm{kHz}$. The drive circuit schematic and the realized circuit is shown in Fig. 5(c) and (d), respectively. The inductors were built in-house using AWG 41 litz wire.

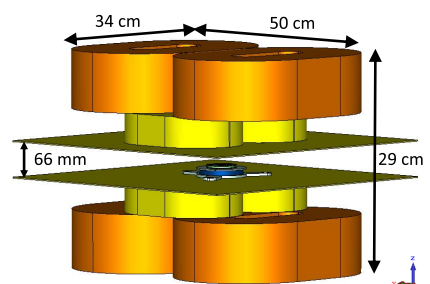

(a)

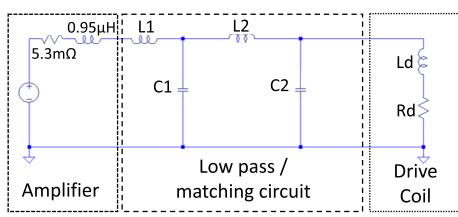

(c)

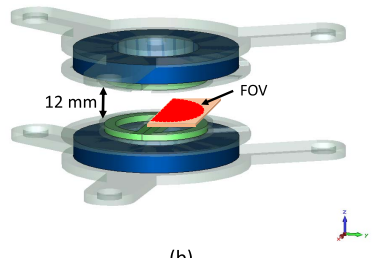

(b)

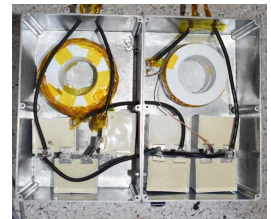

(d)
Fig. 5. (a) The model of the prototype coil system showing the selection, drive/focus, and receive coil placements together with the copper shield. (b) Drive/focus coil and gradiometric receive coil placement. Scanned FOV of $34 \times 18 \mathrm{~mm}^{2}$ and semicircular sensitive region is shown. (c) Drive/focus coil low pass filter and matching circuit model. (d) Realized filter and matching circuit.

The magnetic field of the drive/focus coil was measured at $26 \mathrm{kHz}$ using an inductive pick-up coil $(6 \mathrm{~mm}$ diameter and $6 \mathrm{~mm}$ height) made with a 20 strand AWG 48 litz wire. The efficiency of the drive/focus coil is $0.9 \mathrm{mT} / \mathrm{A}$ at $26 \mathrm{kHz}$.

3) Receive Coil: The receive coil consists of two semicircular Helmholtz coils in a gradiometer configuration in the y-direction (Fig. 5(b)). Each semicircular coil consists of 40 turns wound with a 75 strand AWG 46 litz wire. The receive coil was placed inside the drive/focus coils using a 3D printed mechanical support. Spacing between the upper and lower receive coil sections is $12 \mathrm{~mm}$. Measured inductance and resistance of the receive coil at $26 \mathrm{kHz}$ are $351.5 \mu \mathrm{H}$ and $4.6 \Omega$, respectively. The self-resonance frequency of the receive coil is $870 \mathrm{kHz}$. The efficiency of the receive coil was measured as $1.5 \mathrm{mT} / \mathrm{A}$ using the same measurement coil described in the previous section. The sensitive region of the receive coil, defined as the half-sensitivity contour, is a semicircular region with $20 \mathrm{~mm}$ radius.

\section{B. Scanning Configuration}

An imaging FOV of $34 \mathrm{~mm} \times 18 \mathrm{~mm}(x \times y)$ at the central plane $(\mathrm{z}=0)$ was scanned (Fig. 5(b)) using a combined drive and focus current waveform applied to the transmitter coil. We used a single-cycle triangular focus field with $14 \mathrm{mT}_{p p}$ amplitude and $8 \mathrm{~ms}$ duration to translate the FFL in the whole field of view, and a 200-cycle sinusoidal drive field with $8 \mathrm{mT}_{p p}$ amplitude and $26 \mathrm{kHz}$ frequency (Fig. 6). The distance between upper and lower SF coils were $70 \mathrm{~mm}$. The magnetic field gradient was $G=0.6 \mathrm{~T} / \mathrm{m}$ for this distance. The FFL was rotated from 0 to 177 degrees with 3 degree steps. At each FFL angle, the signal was acquired multiple times (10 or 20 for imaging experiments, 40 for system matrix acquisition), and averaged. We used an $8 \%$ duty cycle $(100 \mathrm{~ms}$ pulse repetition time) to prevent drive coil system from heating. There was about one second delay between transition from one FFL angle to the next. Therefore, the total acquisition time was: (no. of averages $\times 0.1+59$ ) seconds. 

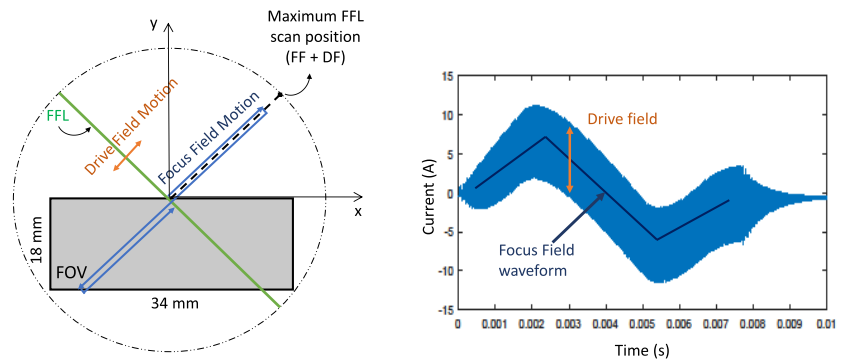

Fig. 6. Left: Trajectory scanned by the FFL by the drive/focus coil, and the field of view. The FFL is at $\phi=45$ in the plot. Right: The measured current waveform applied to the drive/focus coil.

Two reference measurement were taken: before the $0^{\circ}$ scan and after the $177^{\circ}$ scan. The average of these reference scans were subtracted from the data acquired at each angle step. The received coil signal was first amplified (gain $=5$ ) and band-pass filtered (cutoffs: $30 \mathrm{kHz}$ and $300 \mathrm{kHz}$ ) using an SR-560 preamplifier (Stanford Research Systems, MA, USA), then digitized at $2 \mathrm{MHz}$ frequency using an NI-6363 USB (National Instruments, USA) analog to digital converter module. For image reconstruction, we used the system matrix reconstruction method. Details of the reconstruction procedure are given in the next Section.

\section{Image Reconstruction}

For image reconstruction, a regularized optimization problem was solved with the data fidelity constraint, which was outlined in [66]. The system matrix based reconstruction method relies on calibration measurements to construct the system matrix $\mathbf{A} \in R^{m \times N}$, which relates the image $\mathbf{x} \in R^{N}$ to the data $\mathbf{b} \in R^{m}$ as:

$$
\mathbf{A x}+\mathbf{n}=\mathbf{b},
$$

where $\mathbf{n} \in R^{m}$ represents the noise term. Here $N$ is the total number of pixels, and $m$ is the total sample positions.

To obtain the system matrix, an undiluted magnetic nanoparticle (MNP) sample (Perimag, Micromod, Germany) of size $2 \times 2 \times 3 \mathrm{~mm}^{3}$ was scanned inside the $34 \times 18 \mathrm{~mm}^{2}$ FOV in $2 \mathrm{~mm}$ steps $(17 \times 9$ grid size $)$. We used a relatively large sample to reduce the calibration time and increase the SNR at each calibration point. At each position, we gathered a background measurement by taking the sample out of the FOV with a robot $\left(\mathbf{b}_{b c k}\right)$. We then put the sample back into the corresponding position and measured its response for 60 angles of FFL from $0^{\circ}$ to $177^{\circ}\left(\mathbf{b}_{\text {sys }}\right)$. We used 40 times averaging at each scan angle. The acquired signal was transformed into frequency domain using Discrete Fourier Transform.

The reconstruction was done using the Alternating Direction Method of Multipliers (ADMM) that minimizes the $\ell_{1}$-norm and total variation (TV) in the images. As MPI images are naturally sparse and piece-wise continuous, $\ell_{1}$-norm and TV are suitable priors giving superior image quality compared to commonly used algebraic reconstruction technique (ART) and $\mathrm{X}$-space IRadon reconstruction [66], [68], [69].

$$
\begin{aligned}
& \arg \min _{\mathbf{x}} \alpha_{1}\|\mathbf{x}\|_{1}+\alpha_{2} T V(\mathbf{x}) \\
& \text { st. }\|\mathbf{A} \mathbf{x}-\mathbf{b}\|_{2}<\epsilon, \mathbf{x}_{i} \geq 0 \text { for } i=\{1, \ldots, N\} .
\end{aligned}
$$

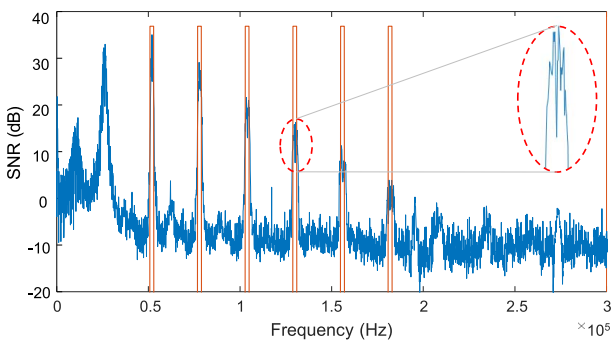

Fig. 7. SNR vs. frequency of the system with calibration phantom placed at the center position. Red line shows the filtered frequency components used for image reconstruction. Harmonics have double peak as a result of low frequency triangular focus field waveform.

Here $\mathbf{x}_{i}$ is the $i^{t h}$ pixel of the vectorized image $\mathbf{x}, \alpha_{1}$ and $\alpha_{2}$ are the weights of the $\ell_{1}$-norm and the TV terms, respectively. We used background corrected measurements $\mathbf{b}=\mathbf{b}_{s y s}-\mathbf{b}_{b c k}$ for reconstruction. The sum of $\alpha_{1}$ and $\alpha_{2}$ is unity. $\epsilon$ is the data fidelity bound. The reader can refer to [66] for the details and algorithm of the ADMM solution procedure. Three basic parameters should be selected carefully for best image reconstruction performance: $\mu, \alpha_{1}$ (or $\alpha_{2}$ ) and $\epsilon$, where $\mu$ is an ADMM specific step size coefficient parameter. Details of parameter selection process is given in the next section.

We calculated the standard deviation of the background signal $\mathbf{b}_{b c k}$, and average signal power per frequency using $\ell_{2}$-norm of the background subtracted signal, $\left\|\mathbf{b}_{s y s}-\mathbf{b}_{b c k}\right\|_{2}$. Figure 7 shows the resultant SNR per frequency for the calibration sample placed at the peak sensitivity position. Note that the harmonics show a double peak due to frequency mixing in the drive/focus current waveform. Although our motivation of using a mixed focus and drive field was to limit the amplitude of the high frequency field, a similar waveform was also shown to improve imaging performance with narrow band high-Q receiver coils [67]. The SNR for a filtered measurement at the FOV center was about $23 \mathrm{~dB}$ before averaging. We used $2^{\text {nd }}$ to $7^{\text {th }}$ harmonics with $2 \mathrm{kHz}$ bandwidth around each harmonic. No significant reconstruction improvement was achieved for a larger bandwidth selection, or by including higher harmonics. This selection resulted in 126 frequency components. Overall number of samples corresponded to $m=7560$ for 60 rotation angles. We then separated the real and imaginary parts of these frequency components, and obtained the system matrix with dimensions $m=15120, N=9 \times 17=153$.

To improve the image reconstruction quality, we applied an upsampling procedure to the acquired system matrix $\mathbf{A}$. We first defined a linear interpolation matrix $\mathbf{D} \in R^{16 N \times N}$ that takes an input image and interpolates it to $4 \times 4$ higher resolution. Then, we used $\mathbf{D}$ matrix along with the system matrix to define the new system matrix $\hat{\mathbf{A}}=\mathbf{A D}^{\mathbf{T}} \in R^{m \times 16 N}$. Hence, the output of the overall reconstruction procedure results in a $0.5 \mathrm{~mm} \times 0.5 \mathrm{~mm}$ pixel size (total $68 \times 36$ pixels), instead of $2 \mathrm{~mm} \times 2 \mathrm{~mm}$ pixel size (total $17 \times 9$ pixels) in the images. Although this procedure does not add any new information in terms of data fidelity, it does not correspond to simple interpolation of the image, as well. Since we use non-linear regularizers such as $\mathrm{TV}$ and $\ell_{1}$-norm, the objective function we impose may improve resolution. We compared 
the result of this upsampling method with interpolation after image reconstruction in the Supplementary Material (Supp. Mat.).

For computational efficiency, we used singular value decomposition (SVD) to downsize the system matrix $\hat{\mathbf{A}}$ :

$$
\hat{\mathbf{A}}=\mathbf{U S V}^{\mathbf{H}} \text {. }
$$

We then modified the data fidelity term as:

$$
\left\|\mathbf{U}^{\mathbf{H}} \hat{\mathbf{A}} \mathbf{x}-\mathbf{U}^{\mathbf{H}} \mathbf{b}\right\|_{2}<\hat{\boldsymbol{\epsilon}} .
$$

By this way, we project the data vector $\mathbf{b}$ onto our system matrix $\hat{\mathbf{A}}$. We also used whitened data and system matrix to include noise statistics in the reconstruction [70]. Details of the whitening process is given in the Supplementary Material. We pre-calculated $\mathbf{U}^{\mathbf{H}} \hat{\mathbf{A}}$ and $\mathbf{U}^{\mathbf{H}} \mathbf{b}$ to speed up the reconstruction. Using these optimizations, each iteration of the algorithm took 0.5 milliseconds on a computer with a single GTX 1080 TI graphical processing unit. 1000 iterations were run for image reconstruction, resulting in 0.5 second reconstruction time from data acquisition to image visualization.

\section{ADMM Parameter Selection}

The ADMM step size coefficient $(\mu)$ was selected large enough to ensure convergence. For fast convergence, we roughly scaled the value of $\mu$ with the average signal intensity for each scenario. Different techniques can be used, such as iteratively changing $\mu$ at each iteration or adaptive ADMM to speed up the reconstruction process [71].

For $\epsilon$ selection, we used the following rule based on the statistics of system matrix and actual measurements: $\epsilon=$ $\max \left\{\kappa, \gamma \times\left\|\mathbf{U}^{\mathbf{H}} \mathbf{b}\right\|_{2}\right\}$. Here, $\kappa$ is calculated from multiple acquisitions for noise estimation, and $\gamma$ is calculated using the high SNR system matrix measurements. For brevity, the details and reasoning behind this selection is provided in the Supplementary Material.

Lastly, $\alpha_{1}$ selection has a high impact on the reconstructed images. In general, $\alpha_{1}$ should be low for large structures, and high for smaller structures to emphasize $\ell_{1}$-norm. Accordingly, we decreased the value of $\alpha_{1}$ in the dot phantom experiments based on dot radius.

\section{E. Imaging Phantoms}

Three different types of phantoms with diluted Perimag nanoparticles were imaged using the open-sided FFL MPI scanner: cylindrical tube phantoms with different tracer concentrations; three-dot phantoms with different dot diameters; and stenosis phantoms. The phantoms were placed inside the FOV using a robot arm.

Four cylindrical tube phantoms were prepared with 1:10 $(772 \mu \mathrm{g} / \mathrm{ml} \mathrm{Fe}), 1: 20(405 \mu \mathrm{g} / \mathrm{ml} \mathrm{Fe}), 1: 100(84 \mu \mathrm{g} / \mathrm{ml} \mathrm{Fe})$, and $1: 750(11 \mu \mathrm{g} / \mathrm{ml} \mathrm{Fe})$ dilution ratios to investigate the performance of the imaging system for different MNP concentrations. Diluted MNP samples were placed inside a $4 \mathrm{~mm}$ diameter polyvinyl chloride (PVC) tube with $20 \mathrm{~mm}$ length. The tubes were filled up to $10 \mathrm{~mm}$ height, and sealed with a sealing film (Supp. Mat.: Fig. 7). 20 averages were acquired at each FFL angle. The acquisition time was 3 minutes.

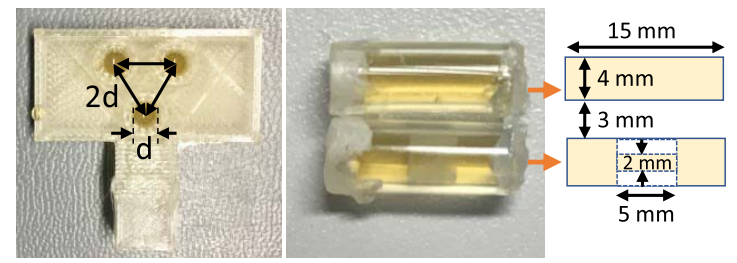

Fig. 8. The photographs of the three-dot phantom (left), healthy vessel phantom (upper middle), and the stenosis vessel phantom (lower middle). Vessel phantom dimensions are shown at the right.

For resolution analysis, phantom housings with three circular holes made of tough polylactic acid (PLA) material were produced using a 3D printer (Ultimaker 3, Ultimaker, Netherlands) (Fig. 8). The holes had $2.5 \mathrm{~mm}, 3 \mathrm{~mm}, 3.5 \mathrm{~mm}$, and $4 \mathrm{~mm}$ diameter, and $3 \mathrm{~mm}$ depth. The distance between the centers of the holes were twice the hole diameter. Ten averages were taken at each FFL angle, resulting in two minutes data acquisition time for each phantom.

Furthermore, a pair of cylindrical phantoms representing healthy and blocked vessels were constructed using PVC tubes (Fig. 8). These phantoms had $4 \mathrm{~mm}$ lumen size and $15 \mathrm{~mm}$ length. The vessel diameter was chosen to mimic human coronary and carotid arteries [72]. The stenosis phantom had a $5 \mathrm{~mm}$ long hollow cylindrical insert $(4 \mathrm{~mm}$ outer, and $2 \mathrm{~mm}$ inner diameter) made of 3D printed tough PLA material. The vessel phantom pair was imaged side by side with a $3 \mathrm{~mm}$ distance between their inner walls. Ten averages were taken at each scan angle.

\section{REsults}

\section{A. Field Properties of the MPI Prototype}

The magnetic field gradient of the $C x$ and $C y$ coil groups was measured at different vertical gap sizes between $40 \mathrm{~mm}$ to $100 \mathrm{~mm}$ (Supp. Mat.: Fig. 1(a)). As the gap size is increased from $40 \mathrm{~mm}$ to $100 \mathrm{~mm}, C x$ coil group gradient efficiency decreases from $8 \times 10^{-3} \mathrm{~T} / \mathrm{m} / \mathrm{A}$ to $5 \times 10^{-3} \mathrm{~T} / \mathrm{m} / \mathrm{A}$, whereas $C y$ coil group efficiency decreases from $3.7 \times 10^{-3} \mathrm{~T} / \mathrm{m} / \mathrm{A}$ to $2.5 \times 10^{-3} \mathrm{~T} / \mathrm{m} / \mathrm{A}$.

The gradient along the FFL was calculated using the measurements at $0^{\circ}\left(C x\right.$ coils excited only) and $90^{\circ}(C y$ coils excited only) rotation angles. The degradation of the gradient is smaller than $10 \%$ along $60 \mathrm{~mm}$ length for the $C x$ coils, and $80 \mathrm{~mm}$ length for the $C y$ coils (Supp. Mat.: Fig. 1(b)).

To demonstrate FFL rotation with the current configuration, the magnetic field in the central imaging plane was measured for the rotation angle of the FFL from 0 to 165 degrees in 15 degree steps (Supp. Mat.: Fig. 2). Magnetic field distributions for FFL translation at $0^{\circ}, 45^{\circ}$, and $90^{\circ}$ rotation angles are shown in the Supplementary Material. Furthermore, translation of the imaging plane in the z-direction ( $z=-15 \mathrm{~mm}, z=0 \mathrm{~mm}$, and $z=15 \mathrm{~mm}$ planes) by asymmetrical excitation of the upper and lower coil groups is also demonstrated in Supp. Mat.: Fig. 5, suggesting that the magnetic field properties are suitable for 3D imaging.

We calculated the sensitivity map of the system using all the frequency components used for image reconstruction (Supp. Mat.: Fig. 6). As a result of gradiometric receive coil design, 

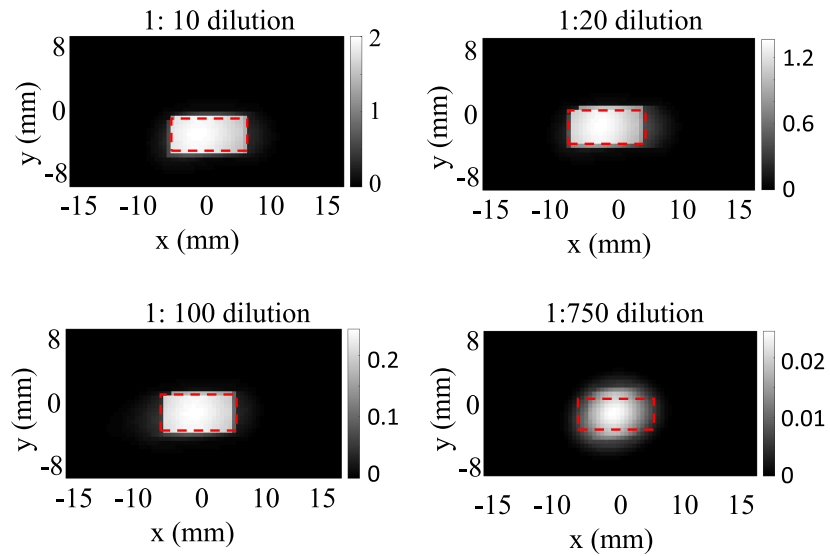

Fig. 9. Imaging results of the cylindrical tubes with different tracer dilution factors. The reconstruction parameters are: $\left(\mu=3, \alpha_{1}=0.5, \epsilon=4.58\right)$, $\left(\mu=3, \alpha_{1}=0.5, \epsilon=2.84\right),\left(\mu=25, \alpha_{1}=0.5, \epsilon=2.1\right)$, and $(\mu=70$, $\left.\alpha_{1}=0.5, \epsilon=2.1\right)$ for $1: 10,1: 20,1: 100$, and 1:750 dilution respectively.

sensitive region is D shaped. Interested readers may also refer to the supporting video showing the sensitivity map at different frequencies around the second harmonic for all FFL angles.

\section{B. Phantom Measurements}

Figure 9 shows the resultant images of the tube phantoms with different tracer concentrations. The coefficients of the $\ell_{1}$-norm $\left(\alpha_{1}\right)$ and TV $\left(\alpha_{2}\right)$ terms were selected to give the best visual image quality. For $\epsilon$ selection, we set a minimum bound based on the average noise of the undiluted calibration sample at the center position (i.e. set $\gamma=0.0675$ ). For the highly diluted phantoms, we observed that $\kappa=2.1$ provides best image quality. Overall, we set $\epsilon=\max \{2.1,0.0675 \times$ $\left.\left\|U^{H} b\right\|_{2}\right\}$

In three-dot phantom experiments, two different tracer concentrations were used. For dot diameters $3.5 \mathrm{~mm}$ and $4 \mathrm{~mm}$, we used 1:100 dilution ratio. On the other hand, for $2.5 \mathrm{~mm}$ and $3 \mathrm{~mm}$ diameters, 1:20 dilution ratio was used, since the dots were not resolvable for 1:100 dilution. Before image reconstruction, we calculated the measured SNR for each sample to find the optimal reconstruction parameters. The SNR for $4 \mathrm{~mm}, 3.5 \mathrm{~mm}, 3 \mathrm{~mm}$ and $2.5 \mathrm{~mm}$ diameter phantoms were $1.76 \mathrm{~dB}, 0.16 \mathrm{~dB}, 9.08 \mathrm{~dB}, 7.11 \mathrm{~dB}$, respectively. We used $\mu=1$ for all cases. We chose the $\epsilon$ parameter similar to the tube phantom case. However, because we used 10 averaging instead of 20 here, we set $\gamma=0.135$, and $\kappa=2.2$. We gradually increased $\alpha_{1}$ as the dot diameter decreased from $4 \mathrm{~mm}$ to $2.5 \mathrm{~mm}$. The reconstruction results show high resolution separation for all phantom types (Figure 10). $3.5 \mathrm{~mm}$ diameter dots are resolvable at 1:100 dilution, while $2.5 \mathrm{~mm}$ diameter dots are resolvable at 1:20 dilution. It should be noted that although we acquired the system matrix with a $2 \mathrm{~mm}$ tracer sample, we generated the results with $0.5 \mathrm{~mm}$ resolution. The small shifts of the apparent positions of the tracers are due to this mismatch. Moreover, brightness of the dots is inversely proportional to the number of pixels they occupy. This is expected since the total energy received for each dot in the image is related to number of particles at each dot (i.e. approximately equal).
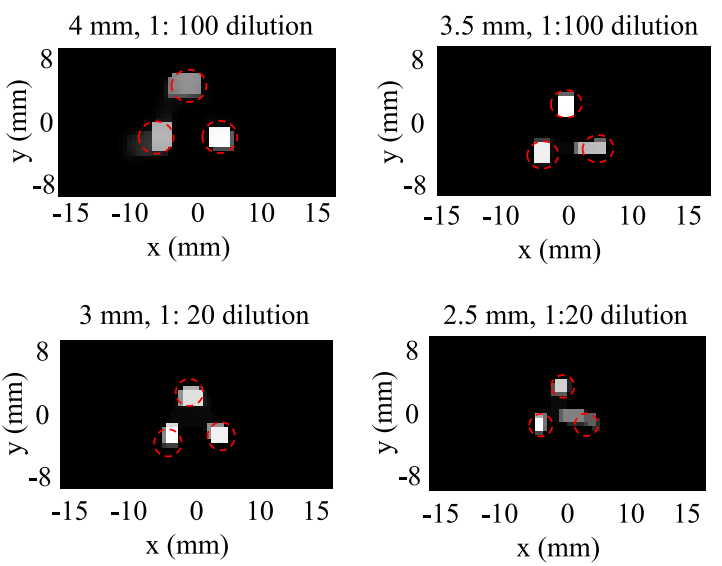

Fig. 10. Imaging results of the three-dot phantoms. For dot diameters $3.5 \mathrm{~mm}$ and $4 \mathrm{~mm}, 1: 100$ dilution ratio was used; whereas for dot diameters $2.5 \mathrm{~mm}$ and $3 \mathrm{~mm}, 1: 20$ dilution ratio was used.The reconstruction parameters are: $\left(\mu=1, \alpha_{1}=0.93, \epsilon=2.2\right),\left(\mu=1, \alpha_{1}=0.985\right.$, $\epsilon=2.2),\left(\mu=1, \alpha_{1}=0.985, \epsilon=3.1\right)$, and $\left(\mu=1, \alpha_{1}=0.99, \epsilon=2.2\right)$ for $4 \mathrm{~mm}, 3.5 \mathrm{~mm}, 3 \mathrm{~mm}$, and $2.5 \mathrm{~mm}$ dot size respectively.

To compare the results with $\ell_{2}$-norm minimization, we also used ART [73] for this phantom type. The results exhibit lower resolution with artifacts compared to the presented reconstruction method (Supp. Mat.: Fig. 8).

The imaging result of the vessel phantom pair is shown in Fig 11. Stenosis is clearly observed in the image, while the healthy phantom shows nearly uniform distribution. The image sizes are in agreement with the actual phantom sizes.

\section{Discussion}

We have shown that a rotating and translating FFL can be generated for MPI using the proposed open-sided configuration. The selection field coils of our prototype system with the present supply configuration can provide a magnetic field gradient of $0.75 \mathrm{~T} / \mathrm{m}$ and $0.5 \mathrm{~T} / \mathrm{m}$ for $40 \mathrm{~mm}$ and $100 \mathrm{~mm}$ vertical gap sizes, respectively. The gradient along the FFL degrades with distance to the FFL center. The FOV of the SF coils is $60 \mathrm{~mm}$ diameter on the xy-plane, if $10 \%$ degradation in the gradient level is allowed inside the FOV.

Single axis drive/focus and receive coils were adequate for 2D imaging. Furthermore, magnetic field measurement results show that slice selection in the coronal plane is also realizable (Supp. Mat.: Fig. 5) to allow 3D imaging by combining multiple slice acquisitions, or by continuously shifting the FFL position along the $\mathrm{z}$-axis. As a result, present configuration has less hardware complexity compared to electronic FFR scanning MPI configurations that require a drive/focus field channel for each imaging axis.

In this work, the imaging time of the total FOV was two to three minutes based on the number of averages. Our drive coil system did not include any means of cooling, which limited the duty cycle of the drive/focus field waveform to $8 \%$, and also hindered continuous rotation of the FFL. Imaging time can be significantly reduced by using improved drive/focus coil(s) that allow high duty cycles.

We used a system matrix reconstruction method that minimizes $\ell_{1}$-norm and total variation in the images. It was shown previously that relatively high resolution images with low 


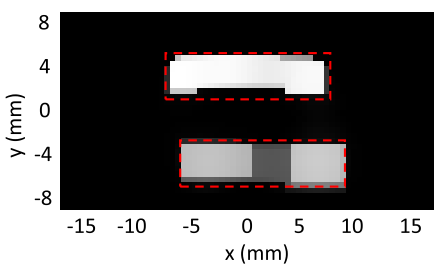

Fig. 11. Imaging result of the phantom pair imitating healthy vessel and vessel with $75 \%$ stenosis (1:100 tracer dilution). The reconstruction parameters are: $\left(\mu=5, \alpha_{1}=0.1, \epsilon=1.92\right)$.

noise can be achieved using these priors for angiographic imaging with MPI [66], [68], [74]. For other applications such as perfusion imaging, these priors may not be directly applicable in the image domain. Nevertheless, other sparsifying transformations or prior information can be considered, which may also improve image quality for perfusion imaging. We used a linear interpolation filter with system matrix to further improve the resolution. With the current hardware, we have achieved reconstruction down to $(11 \mu \mathrm{g} / \mathrm{ml})$ iron concentration, and $2.5 \mathrm{~mm}$ resolution. Using improved receive circuit design e.g. by employing a noise matching network, sensitivities down to nanogram range is achievable [75].

With the use of a gradiometric receive coil, we were able to minimize the transmit-receive coupling and eliminate external noise sources in the measurements without any external shielding. However, the horizontal gradiometer configuration caused a $50 \%$ decrease in the FOV size. Full circular FOV coverage can be achieved with a vertical gradiometer configuration.

Parameters for the reconstruction method we use can be selected with a rigorous parameter selection process. In this work, the parameter $\epsilon$ was selected through statistical analysis accompanied by a fine tuning procedure. $\mu$ parameter was selected to ensure convergence, and $\alpha_{1}$ parameter was chosen by a trial-error approach with a valid reasoning. A further statistical analysis with more data can be performed for the optimum selection of all parameters. For vessel phantoms, it was observed that $\alpha_{1}=0.5$ gives the best results, while for dot phantoms sparsity should be emphasized by choosing greater $\alpha_{1}$. Here let us note that the effect of $\alpha_{1}$ on the reconstructed images is high for this super-resolving scenario. We expect higher robustness in terms of parameter selection for a standard resolution reconstruction [76], [77]. We also note that ADMM allows an adaptive step size $\mu$ that ensures convergence for any initial point [71].

Image resolution in MPI is inversely proportional to the magnetic field gradient. Native resolution, which depends on the SPION magnetization curve, is determined by the particle characteristics as well as selection and drive field parameters [78]. We measured the full width at half maximum (FWHM) resolution of the Perimag particles that we used in our study with an in-house developed relaxometer [79]. For the drive field parameters of our system, the FWHM is about $6 \mathrm{mT}$. This corresponds to a native resolution of about $10 \mathrm{~mm}$ for $0.6 \mathrm{~T} / \mathrm{m}$ gradient. In deconvolution point of view, we can apply deconvolution up to a limit depending on SNR. Modulation transfer function based resolution metric can be used to predict SNR dependent spatial resolution [80]. With the SNR or our system, and using sparsity and TV priors, we were able to get down to $2.5 \mathrm{~mm}$ resolution. High gradient requirement for millimetric resolution is a limiting factor for practical human sized MPI hardware systems. Nevertheless, the use of underlying anatomical structure as a prior for image reconstruction can substantially improve the reconstruction performance. MPI systems combined with MRI [81], [82] or Computed Tomography [83] have already been reported to obtain anatomical information. Our results show that high resolution can be achieved with a relatively low magnetic field gradient using image priors. $75 \%$ stenosis was clearly visible in the 4-mm vessel phantom placed next to the healthy vessel phantom, showing high potential of the present system for cardiovascular applications.

For clinical translation of MPI, magnetic field applied to the body must be within safety limits. The main risks associated with MPI are tissue heating due to absorption of electromagnetic waves (related to the specific absorption rate of the tissues), and peripheral nerve stimulation (PNS). Considering an human torso with $20 \mathrm{~cm}$ radius, bioeffects are PNS dominated below about $40 \mathrm{kHz}$ frequency [84]. We used a relatively low drive field amplitude $\left(8 \mathrm{mT}_{p p}\right)$, and scanned the full FOV with a low frequency triangular wave inside the safety limits. In fact, the use of low drive field amplitude is also advantageous in terms of image resolution [78]. Therefore, the same drive field parameters used in this study can be safely used for a clinical scanner. Nevertheless, the focus field amplitude should be higher than the one used in this work to cover a FOV of clinical size. In addition, a time varying selection field can be used to minimize imaging time. The trajectory of the FFL should be optimized to achieve the best image quality in minimum time within safety limits. Our recent analysis suggest that Uniform Spiral and Radial trajectories may provide a good trade-off between image quality and scan time considering safety limitations [74].

In the current system, total peak power dissipation of the $C x$ and $C y$ coils to generate $0.6 \mathrm{~T} / \mathrm{m}$ gradient on the imaging plane are $0.83 \mathrm{~kW}$ and $7.68 \mathrm{~kW}$, respectively. Therefore, mean peak power dissipation of the selection field coils is $4.26 \mathrm{~kW}$ during an imaging experiment. We previously analyzed the power requirements for a human-sized scanner with similar configuration, which employed soft magnetic material loaded $C y$ coils reduce the power requirement [52]. According to the analyses, a few hundred $\mathrm{kWs}$ of total power is required to cover a $20 \mathrm{~cm}$ diameter $\times 20 \mathrm{~cm}$ height FOV for a $1 \mathrm{~T} / \mathrm{m}$ gradient level, which is practically achievable considering the gradient amplifier technology in MRI.

\section{CONCLUSION}

In this work, we presented the first open-sided FFL MPI prototype scanner to generate tomographic images. The open-sided design allows interaction with the subject for interventional procedures. Adjustable vertical gap size permits the use of highest achievable magnetic field gradient for the imaged subject. Furthermore, 3D imaging is possible with single-axis drive, focus, and receive coils. 2D imaging results have shown that high quality images down to $2.5 \mathrm{~mm}$ resolution can be produced with a relatively low gradient level $(0.6 \mathrm{~T} / \mathrm{m})$, using sparsity and total variation minimization. 
Further studies will focus on 3D imaging with an improved hardware to minimize the imaging time.

\section{ACKNOWLEDGMENT}

The authors would like to thank Dr. Gökçe Kılıç Öger for her help in implementation of the cooling system and cabling of the scanner and Serhat Ilbey for the instrument control setup.

\section{REFERENCES}

[1] R. Weissleder et al., "Superparamagnetic iron oxide: Pharmacokinetics and toxicity," Amer. J. Roentgenol., vol. 152, no. 1, pp. 167-173, Jan. 1989.

[2] Y. X. Wang, S. M. Hussain, and G. P. Krestin, "Superparamagnetic iron oxide contrast agents: Physicochemical characteristics and applications in MR imaging," Eur. Radiol., vol. 11, no. 11, pp. 2319-2331, 2001.

[3] V. F. Cardoso, A. Francesko, C. Ribeiro, M. Ba nobre-López, P. Martins, and S. Lanceros-Mendez, "Advances in magnetic nanoparticles for biomedical applications," Adv. Healthcare Mater., vol. 7, no. 5, 2018, Art. no. 1700845.

[4] O. L. Gobbo, K. Sjaastad, M. W. Radomski, Y. Volkov, and A. Prina-Mello, "Magnetic nanoparticles in cancer theranostics," Theranostics, vol. 5, no. 11, pp. 1249-1263, 2015.

[5] M. Moros et al., "Triggering antitumoural drug release and gene expression by magnetic hyperthermia," Adv. Drug Del. Rev., vol. 138, pp. 326-343, Jan. 2019.

[6] S. Mura, J. Nicolas, and P. Couvreur, "Stimuli-responsive nanocarriers for drug delivery," Nature Mater, vol. 12, no. 11, pp. 991-1003, Nov. 2013.

[7] W. Guo et al., "A magnetic nanoparticle stabilized gas containing emulsion for multimodal imaging and triggered drug release," Pharmaceutical Res., vol. 31, no. 6, pp. 1477-1484, Jun. 2014.

[8] C. Crake et al., "Enhancement and passive acoustic mapping of cavitation from fluorescently tagged magnetic resonance-visible magnetic microbubbles in vivo," Ultrasound Med. Biol., vol. 42, no. 12, pp. 3022-3036, Dec. 2016.

[9] H. Wei et al., "Exceedingly small iron oxide as T1 MRI agents," Proc. Nat. Acad. Sci. USA, vol. 119, no. 9, pp. 2325-2330, Feb. 2017.

[10] C. Rümenapp, B. Gleich, and A. Haase, "Magnetic nanoparticles in magnetic resonance imaging and diagnostics," Pharmaceutical Res., vol. 29, no. 5, pp. 1165-1179, May 2012.

[11] B. Gleich and J. Weizenecker, "Tomographic imaging using the nonlinear response of magnetic particles," Nature, vol. 435, no. 7046, pp. 1214-1217, Jun. 2005.

[12] J. W. Bulte et al., "Quantitative 'hot-spot' imaging of transplanted stem cells using superparamagnetic tracers and magnetic particle imaging," Tomography, vol. 1, no. 2, pp. 91-97, Dec. 2015.

[13] E. Y. Yu et al., "Magnetic particle imaging for highly sensitive, quantitative, and safe in vivo gut bleed detection in a murine model," ACS Nano, vol. 11, no. 12, pp. 12067-12076, 2017.

[14] H. Paysen et al., "Towards quantitative magnetic particle imaging: A comparison with magnetic particle spectroscopy," AIP Adv., vol. 8, no. 5, May 2018, Art. no. 056712.

[15] P. W. Goodwill and S. M. Conolly, "The X-space formulation of the magnetic particle imaging process: 1-D signal, resolution, bandwidth, SNR, SAR, and magnetostimulation," IEEE Trans. Med. Imag., vol. 29, no. 11, pp. 1851-1859, Nov. 2010.

[16] P. W. Goodwill and S. M. Conolly, "Multidimensional X-space magnetic particle imaging," IEEE Trans. Med. Imag., vol. 30, no. 9, pp. 1581-1590, Sep. 2011.

[17] B. Gleich, J. Weizenecker, and J. Borgert, "Experimental results on fast 2D-encoded magnetic particle imaging," Phys. Med. Biol., vol. 53, no. 6, pp. N81-N84, Feb. 2008.

[18] J. Rahmer, J. Weizenecker, B. Gleich, and J. Borgert, "Signal encoding in magnetic particle imaging: Properties of the system function," $B M C$ Med. Imag., vol. 9, no. 1, p. 4, Apr. 2009.

[19] T. Knopp et al., "Weighted iterative reconstruction for magnetic particle imaging," Phys. Med. Biol., vol. 55, no. 6, pp. 1577-1589, Mar. 2010.

[20] P. Vogel, T. Kampf, M. A. Rückert, and V. C. Behr, "Flexible and dynamic patch reconstruction for traveling wave magnetic particle imaging," Int. J. Magn. Part. Imag., vol. 2, no. 2, Nov. 2016, Art. no. 1611001 .

[21] J. Weizenecker, B. Gleich, J. Rahmer, H. Dahnke, and J. J. Borgert, "Three-dimensional real-time in vivo magnetic particle imaging," Phys. Med. Biol., vol. 54, no. 5, p. L1, Feb. 2009.
[22] E. U. Saritas et al., "Magnetic particle imaging (MPI) for NMR and MRI researchers," J. Magn. Reson., vol. 229, pp. 116-126, Apr. 2013.

[23] P. Vogel, M. A. Rückert, P. Klauer, W. H. Kullmann, P. M. Jakob, and V. C. Behr, "First in vivo traveling wave magnetic particle imaging of a beating mouse heart," Phys. Med. Biol., vol. 61, no. 18, pp. 6620-6634, Aug. 2016.

[24] J. Haegele et al., "Magnetic particle imaging: Visualization of instruments for cardiovascular intervention," Radiology, vol. 265, no. 3, pp. 933-938, Dec. 2012.

[25] J. Haegele et al., "Magnetic particle imaging: A resovist based marking technology for guide wires and catheters for vascular interventions," IEEE Trans. Med. Imag., vol. 35, no. 10, pp. 2312-2318, Oct. 2016.

[26] S. Herz et al., "Magnetic particle imaging guided real-time percutaneous transluminal angioplasty in a phantom model," CardioVascular Interventional Radiol., vol. 41, no. 7, pp. 1100-1105, Apr. 2018.

[27] S. Herz et al., "Magnetic particle imaging-guided stenting," J. Endovascular Therapy, vol. 26, no. 4, pp. 512-519, May 2019.

[28] J. Rahmer, D. Wirtz, C. Bontus, J. Borgert, and B. Gleich, "Interactive magnetic catheter steering with 3-D real-time feedback using multi-color magnetic particle imaging," IEEE Trans. Med. Imag., vol. 36, no. 7, pp. 1449-1456, Jul. 2017.

[29] J. Sedlacik et al., "Magnetic particle imaging for high temporal resolution assessment of aneurysm hemodynamics," PLOS ONE, vol. 11, no. 8, Aug. 2016, Art. no. e0160097.

[30] S. Vaalma et al., "Magnetic particle imaging (MPI): Experimental quantification of vascular stenosis using stationary stenosis phantoms," PLoS ONE, vol. 12, no. 1, Jan. 2017, Art. no. e0168902.

[31] S. Herz et al., "Magnetic particle imaging for quantification of vascular stenoses: A phantom study," IEEE Trans. Med. Imag., vol. 37, no. 1, pp. 61-67, Jan. 2018.

[32] B. Zheng et al., "Magnetic particle imaging tracks the long-term fate of in vivo neural cell implants with high image contrast," Sci. Rep., vol. 5, no. 1, p. 14055 , Sep. 2015.

[33] B. Zheng et al., "Quantitative magnetic particle imaging monitors the transplantation, biodistribution, and clearance of stem cells in vivo," Theranostics, vol. 6, no. 3, pp. 291-301, 2016.

[34] J. W. M. Bulte et al., "Developing cellular MPI: Initial experience," in Proc. Magn. Nanopart., Toronto, ON, Canada, vol. 16, Aug. 2010, p. 1675.

[35] J. Dieckhoff et al., "Magnetic particle imaging of liver tumors in small animal models," Int. J. Magn. Part. Imag., vol. 3, no. 2, pp. 1-5. 2017.

[36] E. Y. Yu et al., "Magnetic particle imaging: A novel in vivo imaging platform for cancer detection," Nano Lett., vol. 17, no. 3, pp. 1648-1654, Mar. 2017.

[37] H. Arami et al., "Tomographic magnetic particle imaging of cancer targeted nanoparticles," Nanoscale, vol. 9, no. 47, pp. 18723-18730, 2017.

[38] P. Ludewig et al., "Magnetic particle imaging for real-time perfusion imaging in acute stroke," ACS Nano, vol. 11, no. 10, pp. 10480-10488, Oct. 2017.

[39] X. Y. Zhou et al., "First in vivo magnetic particle imaging of lung perfusion in rats," Phys. Med. Biol., vol. 62, no. 9, pp. 3510-3522, May 2017.

[40] R. Orendorff et al., "First in vivo traumatic brain injury imaging via magnetic particle imaging," Phys. Med. Biol., vol. 62, no. 9, pp. 3501-3509, May 2017.

[41] I. Molwitz et al., "First magnetic particle imaging angiography in human-sized organs by employing a multimodal ex vivo pig kidney perfusion system," Physiol. meas., vol. 40, no. 10, p. 10502, Oct. 2019.

[42] J. Salamon et al., "Visualization of spatial and temporal temperature distributions with magnetic particle imaging for liver tumor ablation therapy," Sci. Rep., vol. 10, no. 1, p. 7480, May 2020.

[43] J. B. Weaver, A. M. Rauwerdink, and E. W. Hansen, "Magnetic nanoparticle temperature estimation: Magnetic nanoparticle temperature estimation," Med. Phys., vol. 36, no. 5, pp. 1822-1829, May 2009.

[44] K. Murase et al., "Usefulness of magnetic particle imaging for predicting the therapeutic effect of magnetic hyperthermia," Open J. Med. Imag., vol. 5, no. 2, pp. 85-99, 2015.

[45] Z. W. Tay et al., "Magnetic particle imaging-guided heating in vivo using gradient fields for arbitrary localization of magnetic hyperthermia therapy," ACS Nano, vol. 12, no. 4, pp. 3699-3713, Apr. 2018.

[46] Z. W. Tay, P. Chandrasekharan, X. Y. Zhou, E. Yu, B. Zheng, and S. Conolly, "In vivo tracking and quantification of inhaled aerosol using magnetic particle imaging towards inhaled therapeutic monitoring," Theranostics, vol. 8, no. 13, pp. 3676-3687, 2018. 
[47] D. Finas et al., "Sentinel lymphnode detection in breast cancer by magnetic particle imaging using superparamagnetic nanoparticles," in Magnetic Nanoparticles: Particle Science, Imaging Technology, and Clinical Applications. Singapore: World Scientific, 2010, pp. 205-210.

[48] C. Z. Cooley, J. B. Mandeville, E. E. Mason, E. T. Mandeville, and L. L. Wald, "Rodent cerebral blood volume (CBV) changes during hypercapnia observed using magnetic particle imaging (MPI) detection," NeuroImage, vol. 178, pp. 713-720, Sep. 2018.

[49] M. Utkur, Y. Muslu, and E. U. Saritas, "Relaxation-based viscosity mapping for magnetic particle imaging," Phys. Med. Biol., vol. 62, no. 9, pp. 3422-3439, May 2017.

[50] M. Möddel, C. Meins, J. Dieckhoff, and T. Knopp, "Viscosity quantification using multi-contrast magnetic particle imaging," New J. Phys., vol. 20, no. 8, Aug. 2018, Art. no. 083001.

[51] J. Borgert, "Perspectives on clinical magnetic particle imaging," Biomed. Eng, vol. 58, no. 6, pp. 551-556, 2013.

[52] C. B. Top, S. Ilbey, and H. E. Güven, "Electronically rotated and translated field-free line generation for open bore magnetic particle imaging," Med. Phys., vol. 44, no. 12, pp. 6225-6238, Oct. 2017.

[53] E. E. Mason, C. Z. Cooley, S. F. Cauley, M. A. Griswold, S. M. Conolly, and L. L. Wald, "Design analysis of an MPI human functional brain scanner," Int. J. Magn. Part. Imag., vol. 3, no. 1, Mar. 2017, Art. no. 1703008

[54] M. Graeser et al., "Human-sized magnetic particle imaging for brain applications," Nature Commun., vol. 10, no. 1, pp. 1-9, Apr. 2019.

[55] J. Weizenecker, B. Gleich, and J. Borgert, "Magnetic particle imaging using a field free line," J. Phys. D, Appl. Phys., vol. 41, no. 10, May 2008, Art. no. 105009.

[56] K. Lu, P. Goodwill, B. Zheng, and S. Conolly, "Multi-channel acquisition for isotropic resolution in magnetic particle imaging," IEEE Trans. Med. Imag., vol. 37, no. 9, pp. 1989-1998, Sep. 2018.

[57] M. Erbe, T. Knopp, T. F. Sattel, S. Biederer, and T. M. Buzug, "Experimental generation of an arbitrarily rotated field-free line for the use in magnetic particle imaging," Med. Phys., vol. 38, no. 9, pp. 5200-5207, Sep. 2011.

[58] K. Bente, M. Weber, M. Graeser, T. F. Sattel, M. Erbe, and T. M. Buzug, "Electronic field free line rotation and relaxation deconvolution in magnetic particle imaging," IEEE Trans. Med. Imag., vol. 34, no. 2, pp. 644-651, Feb. 2015.

[59] G. Bringout et al., "Concept of a rabbit-sized FFL-scanner," in Proc. 5th Int. Workshop Magn. Part. Imag. (IWMPI), Mar. 2015, p. 1.

[60] K. Murase, S. Hiratsuka, R. Song, and Y. Takeuchi, "Development of a system for magnetic particle imaging using neodymium magnets and gradiometer," Jpn. J. Appl. Phys., vol. 53, no. 6, May 2014, Art. no. 067001

[61] P. W. Goodwill, J. J. Konkle, B. Zheng, E. U. Saritas, and S. T. Conolly, "Projection X-space magnetic particle imaging," IEEE Trans. Med. Imag., vol. 31, no. 5, pp. 1076-1085, May 2012.

[62] J. J. Konkle, P. W. Goodwill, O. M. Carrasco-Zevallos, and S. M. Conolly, "Projection reconstruction magnetic particle imaging," IEEE Trans. Med. Imag., vol. 32, no. 2, pp. 338-347, Feb. 2013.

[63] J. J. Konkle, P. W. Goodwill, E. U. Saritas, B. Zheng, K. Lu, and S. M. Conolly, "Twenty-fold acceleration of 3D projection reconstruction MPI," Biomedizinische Technik/Biomed. Eng., vol. 58, no. 6, pp. 565-576, Jan. 2013.

[64] K. Grafe, A. von Gladiss, G. Bringout, M. Ahlborg, and T. M. Buzug, "2D images recorded with a single-sided magnetic particle imaging scanner,' IEEE Trans. Med. Imag., vol. 35, no. 4, pp. 1056-1065, Apr. 2016.

[65] A. Tonyushkin, "Single-sided field-free line generator magnet for multidimensional magnetic particle imaging," IEEE Trans. Magn., vol. 53, no. 9, pp. 1-6, Sep. 2017.
[66] S. Ilbey, C. B. Top, A. Güngör, T. Çukur, E. U. Saritas, and H. E. Güven, "Comparison of system-matrix-based and projection-based reconstructions for field free line magnetic particle imaging," Int. J. Magn. Part. Imag., vol. 3, no. 1, Mar. 2017, Art. no. 1703022.

[67] P. W. Goodwill, G. C. Scott, P. P. Stang, and S. M. Conolly, "Narrowband magnetic particle imaging," IEEE Trans. Med. Imag., vol. 28, no. 8, pp. 1231-1237, Aug. 2009.

[68] M. Storath et al., "Edge preserving and noise reducing reconstruction for magnetic particle imaging," IEEE Trans. Med. Imag., vol. 36, no. 1, pp. 74-85, Jan. 2017.

[69] S. Ilbey, C. B. Top, T. Cukur, E. U. Saritas, and H. E. Guven, "Image reconstruction for magnetic particle imaging using an augmented Lagrangian method," in Proc. IEEE 14th Int. Symp. Biomed. Imag. (ISBI), Melbourne, VIC, Australia, Apr. 2017, pp. 64-67.

[70] T. Kluth and B. Jin, "Enhanced reconstruction in magnetic particle imaging by whitening and randomized SVD approximation," Phys. Med. Biol., vol. 64, no. 12, Jun. 2019, Art. no. 125026.

[71] Z. Xu, M. A. T. Figueiredo, X. Yuan, C. Studer, and T. Goldstein, "Adaptive relaxed ADMM: Convergence theory and practical implementation," 2017, arXiv:1704.02712. [Online]. Available: http://arxiv.org/ abs/1704.02712

[72] J. T. Dodge, B. G. Brown, E. L. Bolson, and H. T. Dodge, "Lumen diameter of normal human coronary arteries. Influence of age, sex, anatomic variation, and left ventricular hypertrophy or dilation," Circulation, vol. 86, no. 1, pp. 232-246, Jul. 1992.

[73] S. Kaczmarz, "Angenaeherte aufloesung von systemen linearer gleichungen,” Bull. Int. Acad. Pol. Sci. Lett. A, vol. 35, pp. 355-357, 1937.

[74] C. B. Top, A. Güngör, S. Ilbey, and H. E. Güven, "Trajectory analysis for field free line magnetic particle imaging," Med. Phys., vol. 46, no. 4, pp. 1592-1607, Apr. 2019.

[75] M. Graeser et al., "Towards picogram detection of superparamagnetic iron-oxide particles using a gradiometric receive coil," Sci. Rep., vol. 7, no. 1, p. 6872, Jul. 2017.

[76] H. E. Guven, A. Gungor, and M. Cetin, "An augmented Lagrangian method for complex-valued compressed SAR imaging," IEEE Trans. Comput. Imag., vol. 2, no. 3, pp. 235-250, Sep. 2016.

[77] E. Kopanoglu et al., "Simultaneous use of individual and joint regularization terms in compressive sensing: Joint reconstruction of multichannel multi-contrast MRI acquisitions," NMR Biomed., vol. 33, no. 4, p. e4247, Jan. 2020.

[78] L. R. Croft et al., "Low drive field amplitude for improved image resolution in magnetic particle imaging," Med. Phys., vol. 43, no. 1, pp. 424-435, Jan. 2016.

[79] C. B. Top, "An arbitrary waveform magnetic nanoparticle relaxometer with an asymmetrical three-section gradiometric receive coil," Turkish J. Electr. Eng. Comput. Sci., vol. 28, no. 3, pp. 1344-1354, May 2020.

[80] T. Knopp, S. Biederer, T. F. Sattel, M. Erbe, and T. M. Buzug, "Prediction of the spatial resolution of magnetic particle imaging using the modulation transfer function of the imaging process," IEEE Trans. Med. Imag., vol. 30, no. 6, pp. 1284-1292, Jun. 2011.

[81] P. Vogel et al., "MRI meets MPI: A bimodal MPI-MRI tomograph," IEEE Trans. Med. Imag., vol. 33, no. 10, pp. 1954-1959, Oct. 2014

[82] J. Franke et al., "System characterization of a highly integrated preclinical hybrid MPI-MRI scanner," IEEE Trans. Med. Imag., vol. 35, no. 9, pp. 1993-2004, Sep. 2016.

[83] P. Vogel et al., "Magnetic particle imaging meets computed tomography: First simultaneous imaging," Sci. Rep., vol. 9, no. 1, p. 12627, Sep. 2019

[84] E. U. Saritas, P. W. Goodwill, G. Z. Zhang, and S. M. Conolly, "Magnetostimulation limits in magnetic particle imaging," IEEE Trans. Med. Imag., vol. 32, no. 9, pp. 1600-1610, Sep. 2013. 